TITLE:

\title{
Video preference assessment and behavioral management of single- caged Japanese macaques (Macaca fuscata) by movie presentation.
}

\section{$\operatorname{AUTHOR}(\mathrm{S}):$}

Ogura, Tadatoshi; Matsuzawa, Tetsuro

\section{CITATION:}

Ogura, Tadatoshi ... [et al]. Video preference assessment and behavioral management of single-caged Japanese macaques (Macaca fuscata) by movie presentation.. Journal of applied animal welfare science 2012, 15(2): 101-112

ISSUE DATE:

2012-03-29

URL:

http://hdl.handle.net/2433/173166

\section{RIGHT:}

(C) Taylor \& Francis Group, LLC; この論文は著者最終稿です。内容が印 刷版と異なることがありますので、引用の際には出版社版をご確認ご 利用ください。This is the Accepted Author Manuscript. Please cite only the published version. 
3 Video preference assessment and behavioral management of single-caged Japanese

11 Running head: Visual preference of Japanese macaques 
Movie presentation can act as an enrichment technique for nonhuman primates,

3 and primates show preferences for certain movie contents over others. This study

4 investigated the video preferences and effects of movie presentation on behavioral

5 abnormalities in single-caged Japanese macaques. When movie rewards were provided

6 as a result of subjects' touch responses, the subjects maintained the touch responses over

7 the course of 40 two-hour sessions. Although the repeated presentation of one stimulus

8 set decreased the touch response of subjects, the replacement of the stimulus set by a

9 new one led to recovery of this response. The subjects showed clear preferences, which

10 were consistent across two different stimulus sets, for movies showing humans or

11 animation (61.1\% of total playing duration). In addition to playing movies with their

preferred content, subjects also consistently played movies with other contents. The availability of a variety of contents might be important for attracting subjects' interest.

14 The frequency with which monkeys engaged in abnormal behaviors decreased in the experimental $(20.9 \%)$ and the post-experimental $(25.6 \%)$ periods compared with the pre-experimental period $(33.5 \%)$. In summary, movie presentations could keep attracting the interest of single-caged monkeys in their visual environment and 
1 ameliorate their behavioral abnormalities for some time. The preference for movies

2 might be influenced by social experience during infancy in Japanese macaques.

3 Keywords: abnormal behavior; animal welfare; Japanese macaque; Macaca fuscata;

4 movie; video preference 
enrichment technique for captive nonhuman primates in some previous studies. Captive presentations of videos, and their sleeping behavior is decreased and locomotion is

$7 \quad$ increased by watching videos (Platt \& Novak, 1997). In experimental situations, movie

8 presentation as a reward was shown to maintain joystick task activities in bonnet

9 macaques (Andrews \& Rosenblum, 1993) and rhesus macaques (Washburn \& Hopkins,

10 1994), suggesting that movie stimuli can attract the interest of captive animals. Thus,

11 movie presentation may be effective as environmental enrichment for captive nonhuman primates.

The content can be assumed to be an important feature of movies used for enrichment techniques and experimental stimuli. In cognitive experiments, primates show different preferences for various still pictures (Sacket, 1966; Redican, Kellicut, \& Mitchell, 1971; Fujita \& Matsuzawa, 1986; Fujita, 1990, 1993; Tanaka, 2003, 2007) and movies (Washburn, Gulledge, \& Rumbaugh, 1997; Andrews \& Rosenblum, 2001; 
1 (2000) reported that movies with varying content provided as environmental enrichment

2 do not differentially affect the behavior of chimpanzees. Thus, the contents of visual

3 stimuli have been shown to cause differing results depending on the particular

4 enrichment or cognitive study. Some biomedical research and zoo management protocols such as those for

6 medical treatment or quarantine require that nonhuman primates be housed individually,

7 although nearly all nonhuman primate species are highly social animals. For their

8 welfare, special care should be devoted to individually housed nonhuman primates.

9 Individually housed chimpanzees watch movies more than the socially housed

10 chimpanzees (Bloomsmith \& Lambeth, 2000). Social stimuli presented through colorf

11 movies are able to induce social responses (Plimpton, Swartz, \& Rosenblum, 1981), suggesting that movie presentation may be a partial substitute for social visual stimulation for captive nonhuman primates.

Movie presentation can be expected to improve animals' behavioral abnormalities, especially in the case of single-caged monkeys, because of the

16 advantages of movie presentations, which include movement as well as color, enabling

17 movies to reproduce multiple features of the complex real visual world such as social

18 stimulation (D’Eath, 1998; Morimura \& Matsuzawa, 2001). This study investigated the 
1 visual preference of single-caged Japanese macaques by presenting movies in a way in

2 which subjects were able to select the played contents. In order to evaluate the movie

3 presentation as an enrichment technique, the effects on the subjects' abnormal behaviors

4 were also investigated.

5

6

$7 \quad$ Subjects

The subjects were three male Japanese macaques (Macaca fuscata) named

9 Romio, Tim, and Sabu at the Primate Research Institute of Kyoto University. They were raised by human caretakers because of their mother's death or rejection. Romio (seven

11 years and six months old when the experiment was started) was hand-reared from birth.

12 Tim (eight years and one month old) was reared by his biological mother at first; however, he was hand-reared by his caretakers after the age of three months. Sabu

14 (approximately two years old) was born in the wild. Starting a few months after his birth, he was protected and reared by human caretakers because he was found to be alone and emaciated. Detailed accounts of the subjects' history are given elsewhere (Murai,

17 Tomonaga, Kamegai, Terazawa, \& Yamaguchi, 2004; Murai \& Tomonaga, 2009). At the 
$1 \mathrm{~cm}$ ) for more than one year. During the daily housing situation and this experiment, the

2 monkeys could see other monkeys in the same room. The monkeys were fed monkey

3 pellets daily and sweet potatoes three times a week, at about $1000 \mathrm{hr}$. They could drink

4 water ad libitum. Routine care of monkeys and experiments were performed in

5 accordance with the guidelines of the Primate Research Institute, Kyoto University.

6 Apparatus

7

8 touch-sensitive display $(30.4 \times 22.8 \mathrm{~cm})($ model L352T-C-BK, Eizo Nanao, Ishikawa,

9 Japan) was attached to each monkey's home cage. The monkeys were allowed to touch

10 the display through the bars of their cages. The apparatus was attached only during the

11 experiment and was removed at other times.

A computer-controlled touch-sensitive display was used in this study. A Stimuli

The stimuli were $21.1 \times 14.2 \mathrm{~cm}$ digitized color movies $(720 \times 480$ pixels, MPEG 1 - Layer 2 files). They did not include sound. The duration of each movie was 10 seconds. Each stimulus set was composed of six categories of content: Humans, Animations, Japanese macaques, Rhesus macaques, Chimpanzees, and Controls (Figure 1). Each category consisted of 96 different movies. They were divided into two stimulus sets: Set 1 and Set 2. Each stimulus set consisted of 288 movies. The humans in the 
1 movies were familiar persons (such as caretakers or researchers) or novel persons. Each

2 of them wore some clothes that were familiar and some that were novel for the subjects.

3 The behaviors of the humans in the movies were walking, working, and conversation

4 with another human. Movies of two familiar humans and two novel humans were used

5 in the first stimulus set (Set 1), and the movies of the same two familiar humans and

6 two different novel humans were used in the second stimulus set (Set 2).

7 Computer-generated moving objects (ball, cube, cone, and so on) and humans were

8 used as the animation movies. The backgrounds of the objects were plain vivid colors.

9 Set 1 consisted of 24 movies of a moving object and 24 movies of a moving human, and

10 Set 2 consisted of 48 movies of a moving human. The movies of Japanese macaques,

11 rhesus macaques, and chimpanzees were filmed in captive enclosures. The behaviors of the animals in the movies were resting, feeding, locomotion, and interaction with other individuals. The individuals in the movies were different between the stimulus sets. The movies of controls were scenery with no animals in Set1 and blinking color in Set 2. 
2 The sessions started between 1400 and $1630 \mathrm{hr}$ and continued for two hours. During the

3 sessions, subjects were allowed to touch the display and to watch the movies. Each

4 subject experienced 40 sessions. Set 1 was used in the first 20 sessions and Set 2 was

$5 \quad$ used in the second 20 sessions.

The experiment was carried out under a sensory reinforcement paradigm with a

7 conjugate reinforcement condition (Rovee-Collier \& Gekoski, 1978; Matsuzawa,

8 1981; Fujita \& Matsuzawa, 1986). A session began with the appearance of a starting

9 stimulus (a red, blue, or green square, $3.7 \times 3.7 \mathrm{~cm}$ ) at a random position on the

10 display. After the subject touched the starting stimulus, a beep sound was played and

11 the starting stimulus disappeared. One second after that, six thumbnails $(8.8 \times 5.9 \mathrm{~cm})$

12 of movies, each of which was randomly selected from one category, were presented

13 randomly within a two-row by three-column matrix on the display. After the subject

14 touched a thumbnail, the selected movie was played in the center of the display. If the

15 subject had made no touch for 10 seconds although the thumbnails appeared on the

16 display, the thumbnails disappeared and the starting stimulus was shown. The selected

17 movie was played as long as the subject touched its thumbnail. If the subject had not

18 touched a thumbnail for three seconds, the beep sound was played and the movie 
1 disappeared. One second after that, the start stimulus was presented at a random

2 position on the display again. When the movie reached its end, it was replayed from

3 the beginning. The duration that each movie was played by a subject was recorded and

4 analyzed as the index of the preference for the content of that movie.

In order to evaluate the effect of the experiment described above on abnormal

6 behavior, the behavior of subjects was compared among the experimental and

7 non-experimental periods. The behaviors of subjects were recorded using a video

8 camera in the following three periods: the pre-experimental period, experimental

9 period, and post-experimental period, for three days. During the observation period,

10 the subject was subjected to the experiment described above. The sessions started at

11 approximately $1400 \mathrm{hr}$ and continued for two hours (i.e., "the experimental period").

12 In addition to this period, the behavior of the subjects during the two hours before the

13 experimental period (i.e., "the pre-experimental period") and two hours after the

14 experimental period (i.e., "the post-experimental period") was also recorded. During

15 the pre- and post-experimental periods, the apparatus was not attached to the cage of

16 the subject. Videotaped behaviors were sampled by using an instantaneous sampling

17 technique (Altmann, 1974). The sampling interval was one minute, and therefore each

18 period included 360 sampling points over a three-day period. We recorded whether the 
1 subject engaged in abnormal behaviors at the sampling points. The definitions of

2 abnormal behaviors were based on Mallapur and Choudhury (2003) and Walsh,

3 Bramblett, and Alford (1982) (Table 1).

4

6

$7 \quad$ Statistical Analyses

Table 1 about here

The total duration of movie playing was calculated in each session for each

9 subject. In order to investigate the effect of the novelty of the movies, the durations of

10 the movie-playing in the first three sessions and the last three sessions for each

11 stimulus set were analyzed using a Generalized Linear Mixed Model (GLMM); the

12 models were constructed using a Poisson distribution. In this analyses, the total

13 durations of movie playing were the response variable. The individuals were contained

14 as random factor. Each data point represented a sum of the duration of movie playing

15 in each session order (the first sessions of Set $1 /$ the last sessions of Set $1 /$ the first

16 sessions of Set $2 /$ the first sessions of Set 2) in each subject. The model which

17 contained the session order and the model without it as the fixed factor were compared.

18 For multiple comparisons of the session order, the models which contained every 
1 combination of the orders as the fixed factors were also compared. As an index of

2 preference for the content, the total playing duration of each content category in each

3 session was calculated. Because some data sets were not normally distributed

4 (Kolmogorov-Smirnov one-sample test had $P<0.05$ ), non-parametric Steel-Dwass'

5 multiple comparisons were conducted on every combination of the six categories in

6 each stimulus set in each subject. The abnormal behaviors during the experimental and

7 non-experimental periods were compared by a GLMM analysis; the models were

8 constructed using a Poisson distribution. In this analysis, the numbers of sampling

9 points at which the subject engaged in abnormal behavior were the response variable.

10 The individuals were contained as random factor. Each data point represented a total

11 number of sampling points in each observation period in each subject. The model

12 which contained the observation period and the model without it as the fixed factor

13 were compared. For multiple comparisons of the observation periods, the models

14 which contained every combination of the periods as the fixed factors were also

15 compared. These statistical analyses would be informal because the number of samples

16 was small. All statistical analyses were conducted with the freeware package $\mathrm{R}$ ( $\mathrm{R}$

17 Development Core Team, 2008). 
2 Preference for Movie Novelty and Contents

$5 \quad( \pm 93.0)$ seconds for Romio, $172.5( \pm 19.3)$ seconds for Tim, and $356.6( \pm 52.2)$ seconds

6 for Sabu. There was a difference between the playing duration for novel movies and

7 non-novel movies (Figure 2). The Akaike Information Criterion (AIC; Akaike, 1974)

8 can be used to compare models with different numbers of fitted parameters. The model

9 with the lower AIC is preferred. The model including the session order (AIC: 1420)

10 showed lower AIC than the model without it as the fixed factor (AIC: 7559). The

11 likelihood ratio test showed a significant difference between the models $\left(\chi^{2}=6144.5, P\right.$ $<0.001)$. In the multiple comparisons of the session orders, the model including the first sessions of Set 1 and Set 2 at a different level from the last sessions showed the lowest AIC (1419). This means that the durations of movie playing in the last sessions of both stimulus sets were shorter than those in the first sessions and there was no difference between the first sessions of two stimulus sets. 
3 the preference pattern in each stimulus set for each subject. All subjects showed a

4 significant differential preference among movies (Table 2). Romio played human

5 movies for the longest duration in both stimulus sets $(40.6 \%$ in Set 1 and $38.6 \%$ in Set

6 2), whereas Tim played animation movies for the longest duration in both stimulus sets

$7 \quad$ (52.0\% in Set 1 and 58.3\% in Set 2), and Sabu played animation movies for the longest duration in Set $1(54.3 \%)$ and human movies in Set $2(34.9 \%)$.

9 multiple comparisons of the observation periods, the model including the each period at

Table 2 about here

\section{Effect of Movie Presentation on Abnormal Behaviors}

Figure 3 shows the frequency of abnormal behavior per hour in each observation period. The model including the observation period (AIC: 88) showed lower AIC than the model without it as the fixed factor (AIC: 119). The likelihood ratio test showed a significant difference between the models $\left(\chi^{2}=35.1, P<0.001\right)$. In the different levels showed the lowest AIC (88.05). This means that the frequencies of 
1 abnormal behavior were differed among the three observation periods. In the

2 pre-experimental period, frequently observed patterns of abnormal behavior were

3 licking hand (11.7\% of the total sampling points), self-biting (8.3\%), and stereotypic

4 pacing $(7.3 \%)$. These behaviors were decreased in the experimental period (licking

5 hand: $10.4 \%$, self-biting: $7.6 \%$, stereotypic pacing: $0.0 \%$ ) and in the post-experimental

6 period (licking hand: $7.7 \%$, self-biting: $4.4 \%$, stereotypic pacing: $8.1 \%$ ). The other

7 patterns of abnormal behavior listed in Table 1 were rarely observed.

8

9

10

Figure 3 about here

\section{DISCUSSION}

We showed here that movie presentation could keep attracting the interest of single-caged Japanese macaques over the course of many experimental sessions. Under a sensory reinforcement paradigm with a conjugate reinforcement condition, the touch responses of all three subjects enabling them to play the movie stimuli were maintained. Repeated presentation of a stimulus set caused a reduction of the subjects' touch responses. Replacement of the stimulus set increased the touch response used to play 
1 the movie stimuli. These data support earlier findings that the novelty of movie stimuli

2 enhances their effectiveness as reinforcers in bonnet macaques (Andrews \& Rosenblum,

3 2001; Brannon et al., 2004). The monkeys showed a clear preference for novel movies. The contents of movie stimuli affected the video preferences of each subject.

5 Romio played human movies for the longest duration, whereas Tim preferred animation

6 movies and Sabu preferred human and animation movies. These tendencies were

7 consistent across the two different stimulus sets, although the preferred contents were

8 different among the subjects. Their video preferences for human and animation movies

9 might have developed as a result of social experiences during the infancy of the subjects.

10 Using still pictures as experimental stimuli, Fujita (1990, 1993) showed that Japanese

11 macaques reared by rhesus macaques preferred pictures of rhesus macaques rather than

12 pictures of Japanese macaques. In chimpanzees, developmental history was also

13 suggested to affect the visual preference regarding still pictures (Tanaka, 2003, 2007).

14 These previous studies suggested that, in the present study, the subjects' social experience with human caretakers during infancy might have contributed to the

16 development of a preference for movies showing humans and animations of characters that were similar to humans. 
1 subjects showed clear preferences for human and animation movies. The human and

2 animation movies accounted only for $39.93 \%$ (Set 2 for Romio) to $72.66 \%$ (Set 1 for

3 Sabu) of total playing duration. The rest of the content viewed by the subjects consisted

4 of movies showing Japanese macaques, rhesus macaques, chimpanzees, and control

5 content. Providing a variety of contents might have been crucial for maintaining the

6 strong interest in movie-presentation shown by Japanese macaques in this study as

7 compared with the shorter watching duration in previous studies (Platt \& Novak, 1997;

8 Bloomsmith \& Lambeth, 2000).

The abnormal behavior, especially licking hands, self-biting, and stereotypic pacing, of the subjects in the experimental and post-experimental periods decreased

11 compared with that in the pre-experimental period. These decreases of abnormal behavior by movie presentation might have been explained by the availability of the apparatus only during the experimental period. Therefore, it remains possible that the playing behavior might simply have replaced the abnormal behaviors. The movie playing would in that case, however, still have been valuable regarding the animals' welfare, because some kinds of abnormal behavior, such as self-biting, are harmful to animals. The decrease of abnormal behavior in the post-experimental period might have been an aftereffect of movie presentation, which might have relieved the stress of the 
1 subjects in the single cages. In any case, our findings indicate that movie presentation

2 could be a valuable technique to improve the abnormal behavior of the single-caged

3 monkeys. environmental enrichment (Reinhardt, 2010). Some of these objects become less

6 attractive to animals in a very short time or occupy a large space. In contrast, movie

7 enrichment can continue to be attractive for a long period if novel and preferred movies

8 are presented, and it requires little space. In the captive condition, movie presentation

9 can add to the visual environment, which is dynamic and complex in the wild. For the

10 welfare of captive nonhuman primates, movie presentation should be provided in

11 combination with other types of enrichment, such as social, physical, and/or

12 feeding-based ones. Movie presentation can be one element in a comprehensive

13 environmental enrichment plan.

This study showed that movie-playing could be effective for improving the abnormal behavior of macaques in single cages, which are an impoverished environment but are inevitable in some special situations of these animals, such as medical care, quarantine, and so on. The ability to provide environmental enrichment in s single cage is limited by the small space, and single cages are a severely restricted 
1 condition with respect to the sensory environment. Movie presentation is an effective

2 technique to enrich the visual environment of the single cage, and the present study

3 showed that single-caged Japanese macaques had a preference for the content of the

4 movie presentation. They had clear preferences for novel movies and movies showing

5 humans or animation. Altogether, our results indicate that the ability of movie

6 presentation to improve behavioral abnormalities of single-caged monkeys could be

$7 \quad$ enhanced by presenting movies with novel, preferred, and/or various contents.

\section{ACKNOWLEDGMENTS}

This work was financially supported by a JSPS fellowship (20-6611) to T. O.,

11 MEXT grant \#20002001 to T. M., and JSPS-gCOE Programs A06 and D07 of Kyoto

12 University. We wish to thank Drs. Y. Ueno, M. Tanaka, N. Morimura, and M. Tomonaga

13 for their valuable comments. We are grateful to the staff members of the Center for

14 Human Evolution Modeling Research, Primate Research Institute, Kyoto University, for their management of the subjects' health. We also thank various students and staff of the

16 Institute for cooperating in preparing the movie stimuli. 


\section{REFERENCES}

2 Akaike, H. (1974). A new look at the statistical model identification. IEEE Transactions on Automatic Control, 19, 716-723.

Altmann, J. (1974). Observational study of behavior : Sampling methods. Behavioural Brain Research, 49, 227-267.

Andrews, M. W., \& Rosenblum, L. A. (1993). Live-social-video reward maintains joystick task - Performance in bonnet macaques. Perceptual and Motor Skills, $77,755-763$.

Andrews, M. W., \& Rosenblum, L. A. (2001). Effects of change in social content of video rewards on response patterns of bonnet macaques. Learning and Motivation, 32, 401-408.

Bloomsmith, M. A., \& Lambeth, S. P. (2000) Videotapes as enrichment for captive chimpanzees (Pan troglodytes). Zoo Biology, 19, 541-551.

Brannon, E. M., Andrews, M. W., \& Rosenblum, L. A. (2004). Effectiveness of video radiata). Perceptual and Motor Skills, 98, 849-858.

Brent, L., \& Stone, A. M. (1996). Long-term use of televisions, balls, and mirrors as 
2 D'Eath, R. B. (1998). Can video images imitate real stimuli in animal behaviour experiments? Biological Reviews, 73, 267-292.

Dobson, A. J. (2002). An introduction to generalized linear models - second edition. London, England: Chapman \& Hall.

6 Fujita, K. (1990). Species preference by infant macaques with controlled social experience. International Journal of Primatology, 11, 553-573.

Fujita, K. (1993). Development of visual preference for closely related species by infant and juvenile macaques with restricted social experience. Primates, 34, 141-150.

Fujita, K., \& Matsuzawa, T. (1986). A new procedure to study the perceptual world of Primates, 27, 283-291.

Mallapur, A., \& Choudhury, B. C. (2003). Behavioral abnormalities in captive nonhuman primates. Journal of Applied Animal Welfare Science, 6, 275-284.

Matsuzawa, T. (1981). Sensory reinforcement: the variety of reinforcers. Japanese Psychological Review, 24, 220-251. (in Japanese with English summary) troglodytes). Journal of Comparative Psychology, 115, 152-158. 
1 Murai, C., \& Tomonaga, M. (2009) Fear responses of Japanese monkeys to scale

2 models. Journal of Ethology, 27, 1-10.

Murai, C., Tomonaga, M., Kamegai, K., Terazawa, N., \& Yamaguchi, M. K. (2004). Do infant Japanese macaques (Macaca fuscata) categorize objects without specific training? Primates, 45, 1-6.

Platt, D. M., \& Novak, M. A. (1997). Videostimulation as enrichment for captive rhesus monkeys (Macaca mulatta). Applied Animal Behaviour Science, 52, 139-155.

Plimpton, E. H., Swartz, K. B., \& Rosenblum, L. A. (1981). Responses of juvenile bonnet macaques to social-stimuli presented through color videotapes. Developmental Psychobiology, 14, 109-115.

Redican, W. K., Kellicut, M., \& Mitchell, G. (1971). Preferences for facial expressions in juvenile rhesus monkeys (Macaca mulatta). Developmental Psychology, 5, 539-539.

Reinhardt, V. (ed.) (2010). Caring hands: discussion by the laboratory animal refinement \& enrichment forum volume 2. Washington, DC: Animal Welfare Institute.

Rovee-Collier, C. K., \& Gekoski, M. J. (1978). The economics in infancy: a review of conjugate reinforcement. In H. W. Reese \& L. P. Lipsitt (eds.) Advances in child 
development and behavior Vol. 13 (pp. 195-255). New York: Academic Press.

Sackett, G. P. (1966). Monkeys reared in isolation with pictures as visual input Evidence for an innate releasing mechanism. Science, 154, 1468-1473.

Tanaka, M. (2003). Visual preference by chimpanzees (Pan troglodytes) for photos of primates measured by a free choice-order task: implication for influence of social experience. Primates, 44, 157-165.

Tanaka, M. (2007). Development of the visual preference of chimpanzees (Pan troglodytes) for photographs of primates: effect of social experience. Primates, 48, 303-309.

Walsh, S., Bramblett, C. A., \& Alford, P. L. (1982). A vocabulary of abnormal behaviors in restrictively reared chimpanzees. American Journal of Primatology, 3, 315-319.

Washburn, D. A., Gulledge, J. P., \& Rumbaugh, D. M. (1997). The heuristic and motivational value of video reinforcement. Learning and Motivation, 28, $510-520$.

Washburn, D. A. \& Hopkins, W. D. (1994) Videotape versus pellet-reward preferences in joystick tasks by macaques. Perceptual and Motor Skills, 78, 48-50. 
2 FIGURE 1 Examples of one frame of a movie in each content category. (a) Humans; (b)

3 Animations; (c) Japanese macaques; (d) Rhesus macaques; (e) Chimpanzees; (f)

4 Controls.

5

6 FIGURE 2 Mean $( \pm$ SE) of the duration of the movie playing in the first three sessions

7 (S) and the last three sessions (E) in each stimulus set for each subject. S and E were

8 compared using a GLMM analysis, which showed that the durations of movie playing

9 in the last sessions of both stimulus sets were shorter than those in the first sessions.

11 FIGURE 3 The frequency of abnormal behavior per hour in each observation period.

12 GLMM analysis was carried out to compare the data between experimental conditions.

13 The frequencies of abnormal behavior were differed among the three observation

14 periods. 
Figure 1

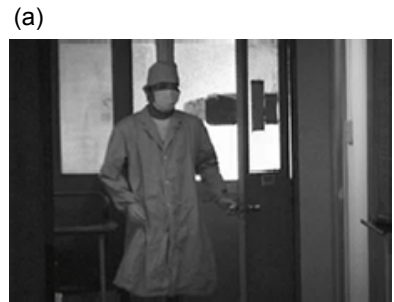

(b)

(c)

(d)
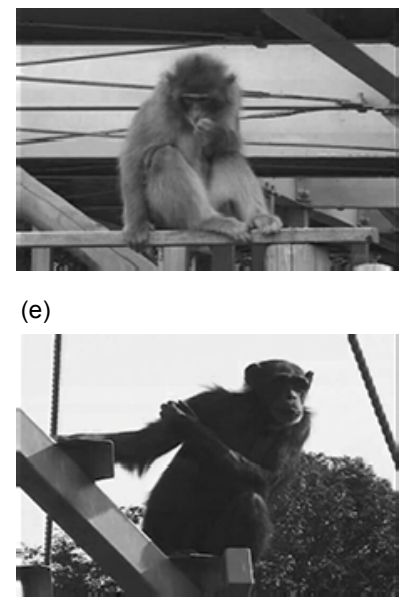

(f)

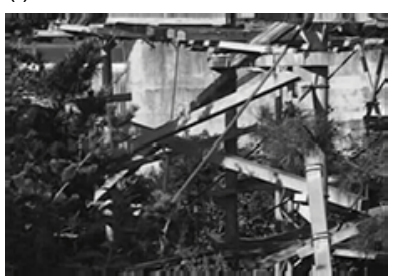


Figure 2

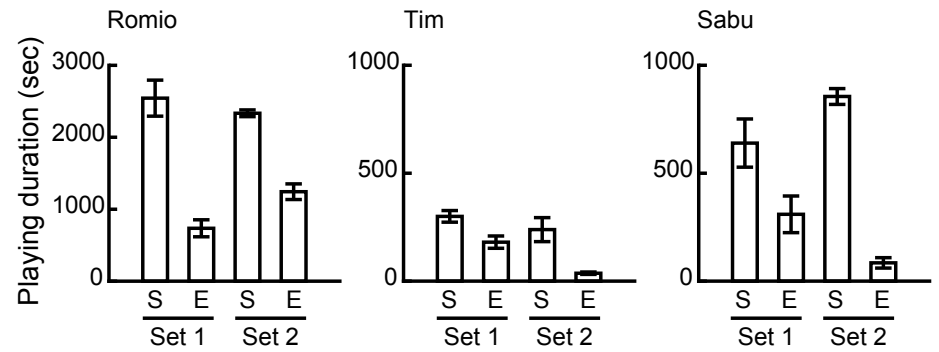


Figure 3

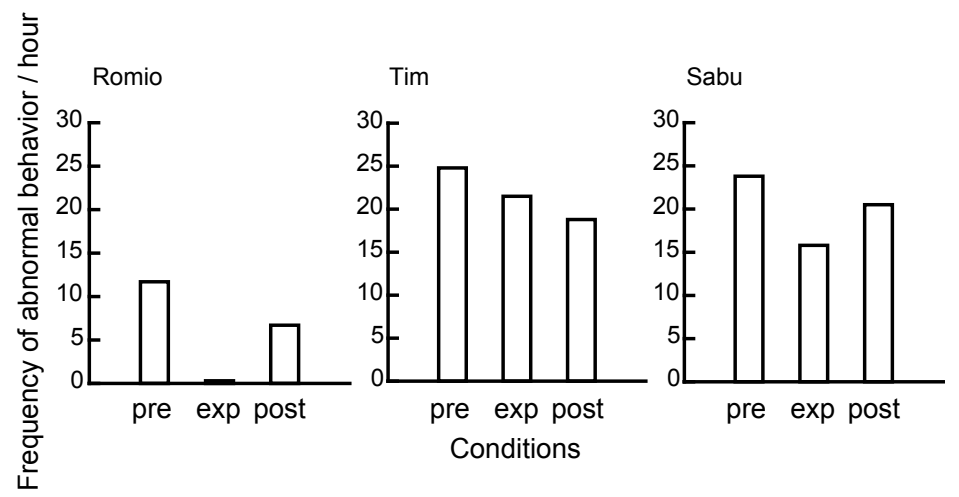


Table 1

List of abnormal behaviors.

Autoerotic stimulation Self-directed sexual activity and masturbation

Floating limb

Unusual movement pattern of a limb; after visually tracking for a

short time, the floating limb is often attacked viciously by oneself

Self-clasping

Use of hands or feet to hold onto part of body by oneself

Self-biting

Hands, legs, arms and/or torso bitten in a stereotyped fashion by

oneself

Stereotypic pacing

Repetitive pacing along the same path

Rocking

Repetitive seated, bipedal, or quadrupedal rocking

Saluting/eye poking

Poking of one or more fingers into the eye

Rubbing lattices

Repetitive rubbing lattices by hands and licking hand

Licking lattices

Repetitive licking lattices

Licking hand

Hands or foot licked by oneself

Hair pulling

Pulling out of own or another animal's hair 
Table 2

Mean ( \pm SE) seconds per session of the playing duration of each content in each stimulus set.

\begin{tabular}{|c|c|c|c|c|c|c|c|}
\hline & & $\mathrm{HU}$ & $\mathrm{AN}$ & JM & $\mathrm{RM}$ & $\mathrm{CH}$ & $\mathrm{CO}$ \\
\hline \multirow[t]{4}{*}{ Romio } & Set 1 & $535.4^{\mathrm{a}}$ & $218.9 \mathrm{~b}, \mathrm{c}$ & $180.5^{b}$ & $194.4^{b}$ & $101.8^{c}$ & $88.5^{\mathrm{c}}$ \\
\hline & & $( \pm 72.7)$ & $( \pm 52.8)$ & $( \pm 20.1)$ & $( \pm 22.5)$ & $( \pm 11.5)$ & $( \pm 10.4)$ \\
\hline & Set 2 & $592.5^{\mathrm{a}}$ & $20.1^{b}$ & $292.7^{\mathrm{c}}$ & $289.3^{c}$ & $165.9 \mathrm{~d}$ & $173.8^{d}$ \\
\hline & & $( \pm 74.1)$ & $( \pm 6.3)$ & $( \pm 35.2)$ & $( \pm 22.0)$ & $( \pm 10.5)$ & $( \pm 16.9)$ \\
\hline \multirow[t]{4}{*}{ Tim } & Set 1 & $39.5^{a}$ & $121.7^{\mathrm{b}}$ & $33.7^{a}$ & $20.2^{\mathrm{a}, \mathrm{c}}$ & $8.0^{c}$ & $10.9^{c}$ \\
\hline & & $( \pm 5.5)$ & $( \pm 18.0)$ & $( \pm 7.2)$ & $( \pm 4.2)$ & $( \pm 1.3)$ & $( \pm 3.1)$ \\
\hline & Set 2 & $9.4^{\mathrm{a}}$ & $64.7^{\mathrm{b}}$ & $11.1^{\mathrm{a}}$ & $17.8^{\mathrm{a}}$ & $1.8^{\mathrm{c}}$ & $6.1^{\mathrm{a}, \mathrm{c}}$ \\
\hline & & $( \pm 2.0)$ & $( \pm 12.1)$ & $( \pm 1.7)$ & $( \pm 3.3)$ & $( \pm 0.6)$ & $( \pm 1.3)$ \\
\hline \multirow[t]{4}{*}{ Sabu } & Set 1 & $75.7 \mathrm{a}, \mathrm{b}$ & $223.4^{\mathrm{a}}$ & $27.2^{\mathrm{c}}$ & $33.8 \mathrm{~b}, \mathrm{c}$ & $25.2^{\mathrm{c}}$ & $26.4^{\mathrm{c}}$ \\
\hline & & $( \pm 13.3)$ & $( \pm 62.1)$ & $( \pm 4.1)$ & $( \pm 5.5)$ & $( \pm 4.6)$ & $( \pm 5.2)$ \\
\hline & Set 2 & $105.1^{\mathrm{a}}$ & 79.6 a, b & $18.1^{c}$ & $49.2 \mathrm{a}, \mathrm{b}$ & $30.8^{b, c}$ & $18.6^{c}$ \\
\hline & & $( \pm 21.0)$ & $( \pm 20.2)$ & $( \pm 2.9)$ & $( \pm 7.4)$ & $( \pm 7.1)$ & $( \pm 5.2)$ \\
\hline
\end{tabular}

Note. Data within a row with different superscripts differ $P<0.05$ in the Steel-Dwass' multiple

comparisons for playing duration of each content in each stimulus set. HU: Humans, AN:

Animations, JM: Japanese macaques, RM: Rhesus macaques, CH: Chimpanzees, CO: Controls. 